\title{
Biologia Futura: Emerging antigen-specific therapies for autoimmune diseases
}

\author{
Gabriella Sármay ${ }^{1}[0$
}

Received: 17 November 2020 / Accepted: 16 January 2021 / Published online: 4 February 2021

(c) The Author(s) 2021

\begin{abstract}
Autoimmune diseases are caused by breaking the central and/or peripheral tolerance against self, leading to uncontrolled immune response to autoantigens. The incidences of autoimmune diseases have increased significantly worldwide over the last decades; nearly $5 \%$ of the world's population is affected. The current treatments aim to reduce pain and inflammation to prevent organ damage and have a general immunosuppressive effect, but they cannot cure the disease. There is a huge unmet need for autoantigen-specific therapy, without affecting the immune response against pathogens. This goal can be achieved by targeting autoantigen-specific $\mathrm{T}$ or B cells and by restoring self-tolerance by inducing tolerogenic antigen-presenting cells (APC) and the development of regulatory T (Treg) cells, for example, by using autoantigenic peptides bound to nanoparticles. Transferring in vitro manipulated autologous tolerogenic APC or autologous autoantigen-specific Treg cells to patients is the promising approach to develop cellular therapeutics. Most recently, chimeric autoantibody receptor T cells have been designed to specifically deplete autoreactive B cells. Limitations of these novel autoantigen-specific therapies will also be discussed.
\end{abstract}

Keywords Antigen-specific $\cdot$ Autoimmune $\cdot$ Diseases $\cdot$ Nanoparticles $\cdot$ Peptides $\cdot$ Therapies

\section{Novelty and innovation}

The main function of the immune system is to differentiate between self and non-self and to destroy pathogenic microbes, while leaving self-tissues intact. The accidental immune response against self is under the control of central and peripheral tolerances, which are influenced by both genetic and environmental factors. The loss of tolerance may lead to the development of autoimmune disorders (Goodnow et al. 2005).

Autoimmune diseases are chronic, and debilitating diseases, sometimes with fatal outcomes, affect about $5 \%$ of the adult population worldwide and are currently incurable. Traditional therapies are not antigen or disease specific, based on general immunosuppressive and anti-inflammatory effect. They can slow down the progression of the disease and even significantly alleviate symptoms but require lifelong treatment and often have life-threatening side effects.

Gabriella Sármay

gabriella.sarmay@ttk.elte.hu

1 Department of Immunology, Eötvös Loránd University, Budapest, Hungary
Additionally, these immunosuppressant therapies make the patients more susceptible to infections and may lead to reactivation of latent pathogens (Rosenblum et al. 2012). Consequently, there is a huge unmet need for the development of antigen-specific, long-term effective tolerogenic therapies.

In the last two decades, biological therapies have emerged in clinical practice representing significant advances over general immunosuppression. These are mostly antibodies or recombinant proteins that selectively suppress different elements of the unwanted immune response to self-antigen, e.g. inhibit of proinflammatory cytokines. Cytokine therapy basically influences memory B cell function, particularly in the post-switch B cell compartment. Biologics such as tumour necrosis factor- $\alpha$ (TNF- $\alpha$ ) inhibitors and interleukin-6 (IL-6) receptor antibodies are considered highly efficacious therapeutic agents. The migratory and homing capacity of B cells might also be altered during cytokine inhibition (Mahmood et al. 2020).

Antigen receptors of autoreactive $\mathrm{T}$ and $\mathrm{B}$ lymphocytes escaping from the control of central and/or peripheral tolerance may target epitopes on multiple self-molecules, some of which are ubiquitously expressed and some are altered by posttranslational modifications. Additionally, due to 
epitope spreading the number of self-epitopes recognized by the immune cells is increasing with disease progression (Vanderlugt and Miller, 2002). Thus, it is exceptionally difficult to identify the ideal epitope(s) that can be applied in autoantigen-specific therapy.

In this minireview, we aimed to summarize the most promising autoantigen-specific therapeutic approaches currently developed and under clinical testing, and we will discuss their advantages and the limitations.

\section{Background}

Autoantigen-specific therapy is based on the strategy called "inverse vaccination", that is to restore unresponsiveness of the immune system towards self-antigen (Passerini and Gregori 2020). Induction of tolerance diminishes self-antigenspecific responses without affecting protective immunity. This can be achieved by manipulating the immunomodulatory function of antigen-presenting cells, deleting or inhibiting self-antigen-specific $\mathrm{T}$ cells or $\mathrm{B}$ cells and by inducing self-antigen-specific regulatory $\mathrm{T}$ cells (Tregs) that have the capacity to suppress the immune response and to induce and maintain the immune tolerance against self.

Two main strategies have been proposed for inverse vaccination: cell-free approaches and cell-based therapies, each of which has limitations (Passerini and Gregori 2020). In cell-free therapies, gene vaccination (antigen-encoding plasmid or viral vectors), antigen-derived proteins/peptides, peptide-MHC complexes, and various antigen-loaded particles (antigen-loaded apoptotic cells or liposomes, antigen-crosslinked erythrocytes or antigen-conjugated/encapsulated nanoparticles) can be applied (Kishimoto and Maldonado 2018). For cell-based therapies, ex vivo induced Treg cells or manipulated tolerogenic dendritic cells (DCs) are exploited. Upon Treg cell therapies, antigen-specific, allospecific or polyspecific Treg cells and chimeric antigen receptor (CAR) Treg cells might be applied (Romano et al. 2019; Roncarolo et al. 2018; Zhang et al. 2018). As dendritic cell therapies, immature tolerogenic DCs, either induced by anti-sense oligos targeting costimulatory molecules or treated with NFkB inhibitor, can be used (Fig. 1).

\section{Cell-based strategies}

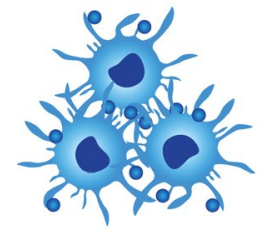

\section{Treg cell therapy}

- Ag-specific Tr1 cellclones

- Allo-specific Tr1 cell lines

- polyclonal Tregs

- CAR-Tregs

- CAAR-T cell

DC cell therapy

- immature toIDC (antisense oligos targeting costimulatory molecules)

- NF-kB inhibitor treated toIDC

\section{Cell free strategies}

\section{Gene vaccination}

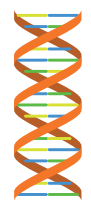

- Ag-encoding plasmids

- Allo-encoding viral vectors

- combined therapies

Proteins and peptides

- whole protein based therapies

(s.c., i.v, i.d, os, i.n.)

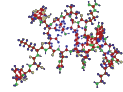

(2)

(native or APLs)

- pMHC-peptide complexes

Vehicles

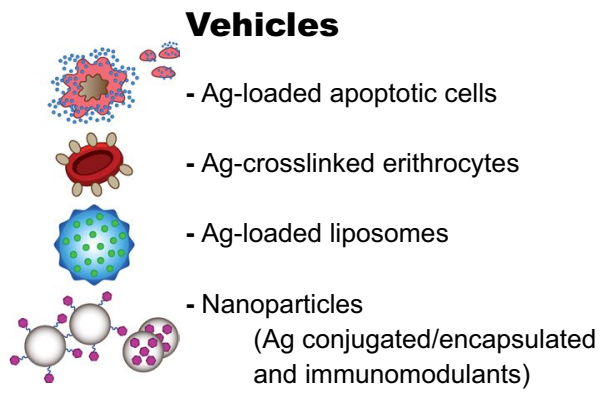

and immunomodulants)

Fig. 1 Cell-free and cell-based strategies to induce antigen-specific tolerance in autoimmune diseases. See text for details. (APL: altered peptide ligand, Treg: regulatory T, CAR: chimeric antigenic receptor, CAAR-T: chimeric autoantibody receptor). Passerini and Gregori (2020) 
Autoreactive B cells play a pathogenic role in many autoimmune diseases; they generate autoantibodies that target different cells and tissues. The B cell antigen receptors (BCR) directly bind antigen and present the intracellularly processed antigenic peptides to T cells; additionally, via their costimulatory molecules they contribute to $\mathrm{T}$ cell activation inducing inflammatory cytokine production. Regulatory B cells (Bregs) also produce the anti-inflammatory cytokines, IL-10 and transforming growth factor beta (TGF $\beta$ ) and express the immune checkpoint molecule, programmed death-ligand 1 (PD-L1) contributing to B cell tolerance (Mauri and Menon 2017). Thus, due to their pathogenic role associated with autoantibody and inflammatory cytokine production, B cells are also considered as therapeutic target in certain systemic autoimmune diseases such as rheumatoid arthritis (RA), myasthenia gravis (MG) and pemphigus vulgaris (PV). Recently, therapeutic approaches focusing mainly on specific depletion or inhibition of autoreactive $B$ cells by genetically modified monoclonal antibodies or by chimeric autoantibody receptor $\mathrm{T}$ cells (CAAR-T) have been proposed (Pozsgay et al. 2016; Lee et al. 2020; Zhang et al. 2020).

\section{Cell-free strategies}

The aim of the inverse gene vaccination is the induction of tolerance to relevant autoantigen by transiently expressing the protein or its epitopes from DNA or RNA vectors in the absence of proinflammatory signals. The coding sequence in DNA vectors is transcribed in the nucleus followed by translation in the cytoplasm and the presentation of the antigenic peptides on MHCI molecules to CD8 + T cells. Professional APC may take up antigens and process as exogenic antigens and present the peptides to CD4 + T cells on MHCII molecules. The activation state of APC has a decisive role regarding the type of response, whether it is immune protection or immune tolerance.

\section{Plasmid DNA}

Intramuscular plasmid DNA delivery is the most often studied, but in case of some vaccine gene delivery to the liver is preferred because of its tolerogenic properties. Induction of tolerance is controlled by several factor, such as the route of administration, target tissue and vector design. This approach was first tested in experimental autoimmune encephalomyelitis (EAE), the mice model of sclerosis multiplex (MS). The plasmid encoding for the myelin basic protein (MBP) prevented the disease. This strategy was also tested in type I diabetes (T1D) model.

Several DNA vaccines were tested in human, e.g. a DNA vaccine encoding MBP in patient with multiple sclerosis, and an insulin-encoding plasmid in patient with type 1 diabetes, albeit with controversial results. Vaccination of newly diagnosed T1D patients with an insulin-encoding plasmid reduced the frequency of insulin reactive $\mathrm{CD} 8+\mathrm{T}$ cells and preserved C-peptide levels (Roep et al. 2013). In a Phase I-II trial, a DNA vaccine encoding MBP was well tolerated and reduced the frequency of cognate TH1 cells in MS patients; furthermore, in Phase II trial it reduced the progression of MS lesions, but failed to reach the primary endpoint (Bar-Or et al. 2007, Garren et al. 2008). In a Phase I trial, a combined effect of a plasmid for T1D antigen and co-encoded TGF$\beta 1, \mathrm{IL}-10$ and IL-2 cytokines is tested. This combination is expected to sustain the antigen-specific Treg induction and thus may lead to stabile tolerance.

Advantages of this technique are the low cost and easy handling; limitations are the activation of endogenous APC and the poor evidence for clinical efficacy. The combination of plasmid DNA with inhibitory cytokines is expected to improve tolerance induction. Ongoing clinical trials will show the value of this approach.

\section{Viral vectors}

Recombinant adeno-associated virus (AAV) vectors and the lentiviral vectors (LVs) are used for targeted gene expression in hepatocytes. The advantage of the viral vectors is the expression of the autoantigen in specific tissues and the avoidance of APC activation. Liver gene therapy with an AAV vector encoding for the myelin oligodendrocyte glycoprotein (MOG) prevented development of EAE and reversed the pre-existing disease by the induction/expansion of antigen-specific FoxP3 + Tregs (Keeler et al. 2018).

Others used LVs to deliver furin-cleavable human insulin to the livers in several animal models of diabetes. This resulted in the reversal of diabetes, restoration of glucose tolerance and pancreatic trans-differentiation (Gerace et al. 2015). The authors concluded that further development is required before the system can be used for gene therapy of T1D.

\section{Protein and peptide delivery}

Repetitive administration of autoantigen in noninflammatory conditions to induce tolerance has been investigated thoroughly on mice models of MS and T1D. The idea behind this is that continually applied high dose of proteins or peptides without proinflammatory adjuvants will be taken up and presented to T cells by tolerogenic APCs and thus may induce deletion or clonal anergy of autoreactive effector $\mathrm{T}$ cells and the differentiation of antigen-specific Tregs (Pozsgay et al. 2017; Zhang et al. 2018).

The first antigen investigated for the protein-based immunotherapy was insulin, the antigenic target in non-obese 
diabetic mice (NOD) model of T1D (Nakayama et al. 2005). After the initial positive results in mice, insulin was added orally or intranasally to T1D patients in different settings (Fourlanos et al. 2011; Bonifacio et al. 2015). None of the trials have achieved the clinically desirable result thus far. Intramuscular injection of Insulin B with incomplete Freund's adjuvant induced InsB-specific Tregs, but did not alter C-peptide level (Orban et al. 2010). Similarly, the long-studied oral tolerization with myelin antigens in MS, although modulated the antigen-specific immune response, showed no therapeutical efficacy (Hafler et al. 1997).

Beside the protein-based approaches, peptide epitopes from autoantigens were also tested both in T1D and in MS. For a successful peptide therapy in the treatment of autoimmune diseases, it is essential to know the major autoantigens responsible for initiating the disease. The presence of autoantibodies and/or antigen-specific $\mathrm{T}$ cells would help to select the disease-relevant peptides that can be applied in therapy. The initial peptide-based trials have not shown clinically relevant results, most probably because autoimmune diseases are triggered by many $\mathrm{T}$ cell clones with different specificity due to epitope spreading (Vanderlugt et al. 2002). Therefore, in recent trials a mixture of peptides from multiple autoantigens has been applied in T1D (MulipepT1De (NCT02620332) and the IMCY-0098 trial (NCT03272269) (Alhadj et al. 2017) and in relapsing MS patients (MBPderived peptide cocktail ATX-MS-1467). In the latter case, an association was found between the treatment and the reduction in MRI lesions (Chataway et al. 2018). NexVax2, composed of three immunodominant gliadin peptides, has been tested in Phase I clinical trial in celiac disease (CD) patients. The treatment was safe and well tolerated, and $\mathrm{T}$ cells did not respond to gluten challenge after the treatment, indicating tolerance induction. A Phase II clinical trial is underway (Yoosuf et al. 2019).

The increasing amount of information on MHC binding and TCR interacting residues of T cell epitopes made the design of altered peptide ligands (APL) possible. APL carried amino acid substitutions at contact positions with MHC or TCR and could trigger altered TCR signalling resulting in different functional outcome. This raised the possibility that APL might modulate autoimmune response by triggering the expansion/induction of Tregs upon peptide recognition (Candia et al. 2016). APLs were tested in MS and T1D patients (Bielkova et al. 2000, Walter et al. 2009), but they induced hypersensitivity and antibody production cross-reacting with the native peptide in several patients, while other APL induced TH1 skewing of CD4 + cells crossreacting with the native peptide or had no effect on T1D. Thus, despite the promising results in mice, clinical trials with APL have not been successful so far.

Since the activation state of the host APC critically influences whether the consequence of peptide recognition will be tolerance or cell activation, one of the strategies to exclude APC is the application of peptide-MHC complexes to directly activate T cells. Vaccination with peptide -MHC complexes without co-stimulation is predicted to induce tolerance. This strategy was tested in preclinical models of experimental autoimmune encephalomyelitis (EAE) (Sinha et al. 2007) and NOD mice (Lin 2010) and resulted in unresponsiveness of $\mathrm{T}$ cells. Further clinical trials are necessary to see if peptide-MHC complexes have therapeutic significance.

\section{Nanoparticle- and cell-based approaches}

Because of the limitations of soluble protein and peptide delivery into the organism to induce tolerance, several strategies were developed to use various particles or cells as vehicles to deliver antigens/peptides to APCs, for direct triggering of Treg cells, as immunoregulatory device, or as $\mathrm{T}$ cell tolerance inducing APCs.

\section{Antigens-/peptides-coupled carrier cells}

A promising strategy is the induction of T cell anergy by using carrier cells coupled to autoantigenic peptides. In this method, autoepitope peptides chemically cross-linked to syngeneic splenocytes or peripheral blood mononuclear cells (PBMC) using 1-ethyl-3-(3-dimethylaminopropyl) carbodiimide (ECDI), which promotes antigen coupling and induces apoptosis of the carrier cells (Getts et al. 2013). Such apoptotic cells are taken up by APC that directly present peptides-MHCII complexes to CD4 + T cells, and in the absence of co-stimulation signal trigger IL-10 and TGF $\beta$ secretion, and the upregulation of PD-L1 resulting in the apoptosis of pathogenic $\mathrm{T}$ cells and the induction of Tregs. The efficacy of this strategy was proven in preclinical models such as EAE and NOD mice (Getts et al. 2013). Furthermore, an MS-related seven-peptide mix coupled to autologous peripheral blood cells was administered into MS patients in a Phase I-II clinical trial. The results have proven the safety and tolerability of the treatment that also reduced the antigen-specific T cell response (Lutterotti et al. 2013).

Antigenic peptides can also be loaded to erythrocytes imitating the tolerization mechanism of dying red blood cells. In this case, the peptides must contain glycophorin A or sortase A binding sequences to fix the peptide to erythrocytes. This approach was found to be effective in EAE and NOD mice and resulted in the depletion of antigen-specific T cells in vivo (Cremel et al. 2013). Applying a similar strategy in a Phase Ib trial, a reduced myelin-specific T cell response and increased frequency of Tregs ( $\operatorname{Tr} 1$ and $\mathrm{mTreg})$ were observed, indicating the induction of immune tolerance (Lutterotti 2019). 


\section{Peptide-loaded nanoparticles}

Fragments of apoptotic cells are taken up by professional APCs in the absence of activation signals that induce tolerance. This mechanism is mimicked by in vitro modified apoptotic spleen or blood cells carrying an autoantigen (Hunter et al., 2014). However, isolating, manipulating and returning autologous cells to the patient is an expensive and cumbersome procedure. Nanoparticles can be synthetic alternatives to autologous apoptotic cells, whose beneficial properties include, in addition to simpler production, their well-defined and reproducible physiochemical characteristics, as well as the precise controllability of their antigen content and its release (Pearson et al. 2019).

Nanoparticles loaded with autoantigenic peptides can specifically target autoreactive cells and induce their anergy or apoptosis, thereby achieving antigen-specific immunosuppression (Pozsgay et al. 2016; Feng et al. 2020). Although immunosuppression-based approaches represent important therapeutic options, the primary goal in the treatment of autoimmune diseases is to prevent the formation of autoreactive effector cells and to restore long-term tolerance to self-antigen. This can be achieved primarily through the presentation of the autoantigen in question in a tolerancepromoting manner. Nanoparticles containing a diseaserelevant autoantigen (and possibly an immune modulator agent) are selectively taken up by antigen-presenting cells (APC) that induce a tolerogenic phenotype of APC, so that the introduced antigen is presented in a tolerogenic manner, resulting in the development of regulatory $\mathrm{T}$ and $\mathrm{B}$ cells (Pearson et al. 2019; Feng et al. 2020). Alternatively, artificial APC (aAPCs) can be fabricated by applying peptide-MHC decorated nanoparticles that directly trigger antigen-specific Tr1-like T cells (Feng et al. 2020). Thus, tolerant nanoparticles (tNPs) act directly or indirectly on lymphocytes and induce the expansion of regulatory cells, the function of which leads to the elimination or inhibition of activation of naïve and effector cells specific for the target antigen (Kishimoto and Maldonado 2018).

For tolerance induction, the use of polymer-based particles is predominant, of which poly-(lactic-co-glycolic acid) (PLGA) biodegradable nanoparticles (NPs) have proven to be the most suitable. The goal is to produce particles that are selectively taken up only by APCs, primarily DCs and macrophages. The accumulation of particles at appropriate sites within the body and their phagocytosis by target cells are primarily influenced by their size, shape and surface charge (Kishimoto and Maldonado 2018). The efficiency of nanoparticle uptake by APCs can be increased by various structural modifications, the most obvious is to fine-tune the surface properties of the particles. Hunter et al. modified the surface of the PLG particles with a poly(ethylene maleic acid) (PEMA) surfactant layer and showed that the
Ag-PLG-PEMA particles carrying surface-conjugated antigen were more efficient to induce tolerance than Ag-PLG particles (Hunter et al. 2014). Ag-PLG-PEMA particles were protective when pre-administered in a mouse model of relapsing-remitting experimental autoimmune encephalomyelitis (R-EAE) and resulted in complete remission and prevention of relapse after induction (Hunter et al. 2014).

The tolerogenic effect of NPs can also be favourably affected if, in addition to the antigen, the particles also contain immunomodulatory agents, such as rapamycin. Rapamycin acts on the mTOR signalling pathway that regulates cellular metabolism, and it is an allosteric inhibitor of mTOR serine-threonine kinase. Administration of rapamycin into the nanoparticles provides selectivity, since only APCs can take them up. Inhibition of the mTOR pathway in these cells initiates autophagic processes, leading to a decrease in the expression of costimulatory molecules, and thus results in a tolerogenic phenotype (Kishimoto and Maldonado 2018).

More recently, NPs coated with MHCI and MHCII molecules in complex with antigenic peptides have been extensively studied in different preclinical models (Serra and Santamaria 2019). Treatment of NOD mice with NPs coated with the prevalent islet glucose 6-phosphatase-related protein (IGRP) epitope (IGRP206-214)-MHCI complexes protected the NOD mice from diabetes, and this was associated with the deletion of the pathogenic high-avidity autoreactive $\mathrm{T}$ cells and the expansion of memory-like $\mathrm{CD} 8+\mathrm{T}$ cells with regulatory properties (Tsai et al. 2010).

Disease-relevant peptide-MHCII NPs were tested in different mice models of T1D, MS and arthritis. These MHCII-peptide-NP complexes consistently reversed the established disease, while induced expansion and differentiation of pathogenic $\mathrm{CD} 4+$ effector $\mathrm{T}$ cells into disease-suppressing TR1 cells, differentiation and recruitment of Bregs, and suppression of antigen presentation (Clemente-Casares et al. 2016, Serra and Santamaria 2019). The peptide-MHCcoated NPs have been validated in several preclinical models of autoimmunity and are currently under clinical tests.

\section{Cell-based therapies}

Cell-based approaches to promote or restore tolerance in autoimmune diseases can lead to control the unwanted immune response. Therapies based on adoptive transfer of regulatory $\mathrm{T}$ cells (Tregs) or dendritic cells (DCs) are now under clinical trials to study the safety and feasibility of the approach.

\section{Treg-based approaches}

Tregs are small but essential subset of lymphocytes which can suppress immune response and maintain immune 
homeostasis by preventing inflammation and development of autoimmune diseases (Sharabi et al. 2018). Tregs in patients with systemic or organ specific autoimmune diseases have lower number and/or impaired function as compared to healthy controls. Thus, expanding the pools of patient's Tregs ex vivo or in vivo is a promising way to restrain autoimmune diseases (Sharabi et al. 2018; Ferreira et al. 2019). Tregs have shown an increasing diversity and are generally divided into two main populations, thymus-derived Treg and peripheral CD4 + Treg cells. Peripheral Tregs are constitutively expressing CD25 and FoxP3 +, ; the latter is necessary for the suppressive functions (Sharabi et al. 2018). Another subset is called regulatory type I $(\operatorname{Tr} 1)$ cells that are characterized by CD49b and LAG3 expression and the ability to produce IL-10 and TGF $\beta$ (Levings et al. 2001, Roncarolo et al. 2018). Treg cells are responsible for dampening autoimmune response; thus, Tregs have been considered as living drugs for autoimmune and inflammatory diseases (Ferreira et al. 2019). CD25 + FoxP3 + Tregs are easy to expand in vitro, and thus they have been used in several clinical trials including the treatment of T1D patients (Battaglia et al. 2006; Roncarolo et al. 2018). The infunded cells remained stable and detectable for about 1 year in some cases, but in other cases the effect was transient and had no therapeutic benefit. This can be explained by the fact that the transferred cells were not selected for antigen specificity and therefore contained a very low number of autoantigen-specific Tregs.

FoxP3 + Tregs constitutively express CD25, the alfa chain of IL-2 receptors and low dose of IL-2, especially if its binding affinity to CD25 is artificially increased and was considered to selectively expand Tregs without affecting $\mathrm{T}$ and NK cells (Abbas et al. 2018). Indeed, low dose of IL-2 has shown an acceptable safety profile and is currently tested in several clinical trials. The in vivo expanded polyclonal Tregs were expected to contain high number of antigen-specific Tregs; however, since the cells were not selected for antigen specificity, the risk of the general immunosuppression may also be true for this situation. A possible solution to avoid broad immunosuppression could be the local application of IL-2.

Polyclonal and antigen-specific Tr1 cells have also been used in clinical trials, but similarly to CD25 $+\mathrm{T}$ cells they had limited survival capacity in vivo (Desreumaux et al. 2012). To overcome this problem, conventional CD $4+T$ cells were generated, that were converted to $\operatorname{Tr} 1$ cells by lentiviral transduction of IL-10 (Andolfi et al. 2012, Gregori et al. 2018). An alternative possibility to solve the issue of antigen selectivity is to develop regulatory chimeric antigen receptor T (CAR-Treg) cells (Fransson et al. 2012, Zhang et al. 2018). In this case, a single-chain variable fragment $(\mathrm{scFv})$ of a monoclonal antibody recognizing myelin oligodendrocyte glycoprotein (MOG) is engineered in a chimeric antigen receptor molecule that expresses intracellular domain of $\mathrm{CD} 28$ and $\mathrm{CD} 3$ molecules. CD4 + T cells were modified by a lentiviral vector system to express CAR in trans with the murine FoxP3 gene that drives Treg differentiation. The stable expression of FoxP3 by genetic engineering blocks Treg conversion into effector cells. The CAR $\alpha M O G$ receptor attaches the Tregs to the vicinity of MOG + oligodendrocytes. The CNS-targeting Tregs efficiently suppressed ongoing inflammation in EAE, the mice model of MS, and diminished disease symptoms (Fransson et al. 2012). CAR-Tregs are promising, if the cells maintain a stable phenotype, and more specific and potent as compared to polyclonal Tregs, but there are several issues to be solved before it can be used in clinic. It is not known that CARTregs can induce adverse reactions like cytokine storm and neuronal cytotoxicity. Moreover, selection of autoantigen to be targeted by CAR and the development of specific antibodies is challenging in some autoimmune diseases. Finally, CAR-Treg exhaustion may limit their therapeutical efficacy (Zhang et al. 2018).

CAR-T cells have also been used recently to specifically target and kill autoantigen-specific B cells. In pemphigus vulgaris (PV), a life-threatening autoimmune blistering disease caused by autoantibodies to the keratinocyte adhesion protein desmoglein 3 (Dsg3), the pathogenic memory B cells express Dsg3-specific B cell receptors (BCRs). A chimeric autoantibody receptor (CAAR) was created using the autoantigen Dsg3 as the CAAR extracellular domain. The CAAR-T-dependent depletion of Dsg3-specific B cells had therapeutic effects in a murine model of PV (Ellebrecht et al. 2016). Recently, preclinical studies were published that have supported an investigational drug application enabling a Phase I trial to evaluate the safety and preliminary efficacy of DSG3-CAAR-T as a targeted precision therapy in patients with anti-DSG3 mucosal PV (Lee et al. 2020).

The main limitation of CAR-T cell therapy is the heterogeneity and complexity of systemic autoimmune diseases: the autoantigen profiles of individual patients are different, and due to the epitope spreading the specificity also varies as the disease progresses. Therefore, to address these issues, a universal anti-fluorescein isothiocyanate (FITC) CAR-T cell has been recently developed that in combination with FITClabelled RA immunodominant citrulline-containing peptide is able to kill patient-derived autoreactive B cells (Zhang et al. 2020). This universal CAR-T cell system provides a direction for customized therapies that might be applied to other systemic autoimmune diseases as well (Fig. 2).

\section{DC-based therapies}

DCs are professional antigen-presenting cells with phenotypic heterogeneity, and they play a critical role in the maintenance of tissue homeostasis and in promoting tolerance, thus acting as regulatory cells (Raker et al. 2015). 
1. Identification of the kinds of autoantibodies

\section{Preparation of universal (anti-FITC) CAR-T cells}

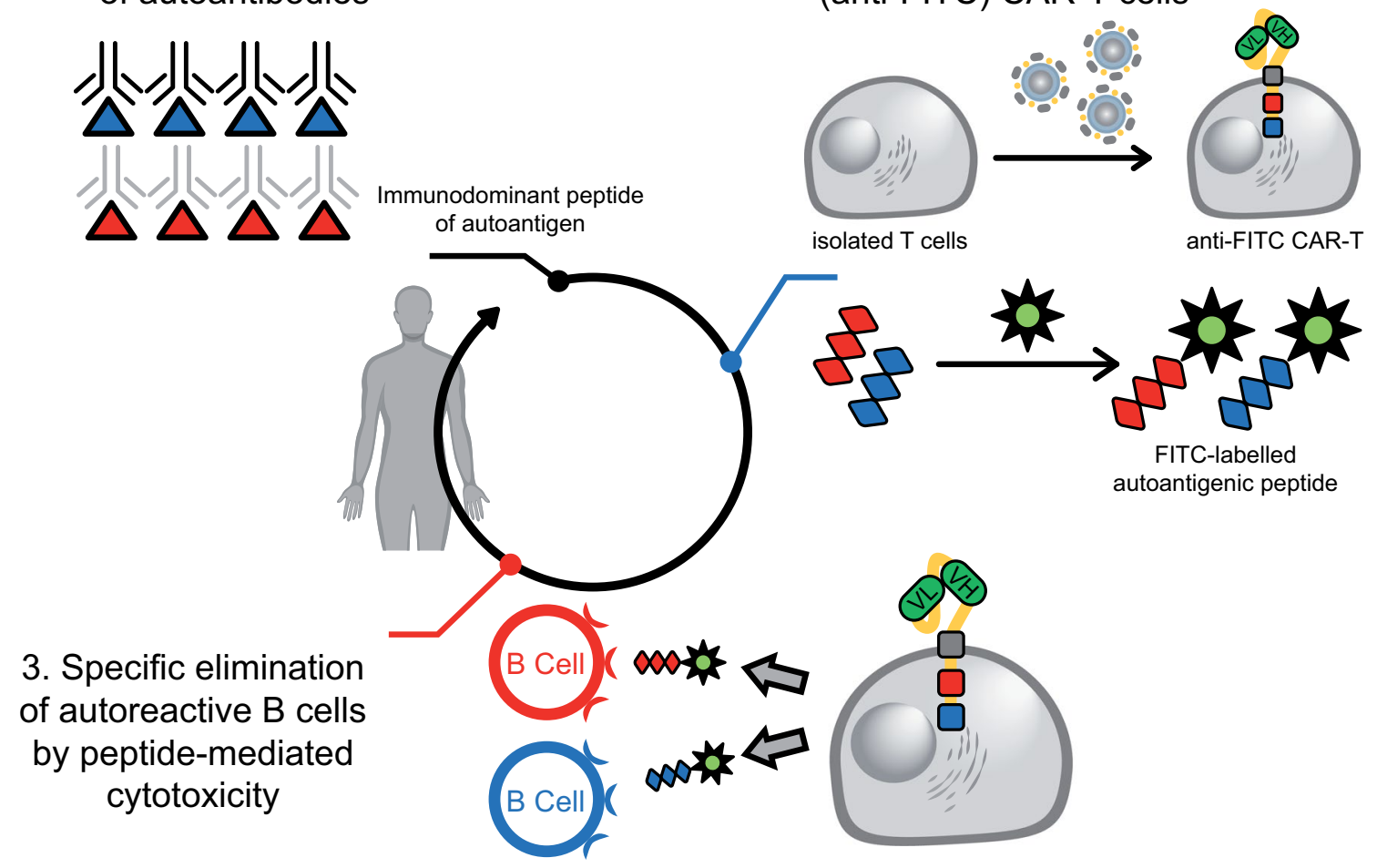

Fig.2 Graphic illustration of the main steps leading to the elimination of autoreactive B cells mediated by universal (anti-FITC)-CAR-T cells and FITC-labelled autoantigenic peptides. (1) Identification of the autoantibody specificity and determination of autoantigenic pep-

The regulatory capacity of DCs depends on their immature state and distinct subsets, yet not restricted to this. The tolerogenicity of DC is controlled by a complex network of environmental signals and cellular intrinsic mechanisms. DCs with regulatory properties are called tolerogenic DC (tolDC); they present antigen to T cells, while downregulating costimulatory molecules and proinflammatory cytokines and upregulating inhibitory/modulatory receptors and anti-inflammatory cytokines, resulting in the induction of antigen-specific Tregs. DC can be differentiated relatively easily from macrophages, and these ex vivo generated DC can be differentiated into tolDC by different manipulations, such as inhibiting NFkb, silencing costimulatory molecules or inducing the expression of immunoregulatory molecules such as IL-10, IL-4 and indoleamine 2,3-dioxygenase (IDO) (Liu et al. 2015). A better understanding of the biology and the in vitro generation of tolDC opened the way for the application of autologous tolDC as immunotherapy in clinical trials for autoimmune diseases (Ten Brinke et al. 2015). Autologous TolDCs generated ex vivo from macrophages can induce, enhance or restore antigen-specific tolerance in vivo by several mechanisms, such as T cell deletion or inhibition, induction of $\mathrm{T}$ cell anergy, generation of tides by ELISA, (2) preparation of anti-FITC CAR-T cells and FITClabelled peptides, (3) elimination of corresponding autoreactive $\mathrm{B}$ cells recognizing the peptide through FITC-peptide-mediated CAR-T cytotoxicity. Zhang et al. (2020)

Tregs and expansion of pre-existing Tregs and modulation of APCs.

Several tolerogenic approaches have been used: in the first-in-man clinical trial, autologous tolDC treated with anti-sense oligonucleotides silencing CD40, CD80 and CD86 to keep their immature state was infused to T1D patients (Giannoukakis et al. 2011). Another study aimed to explore the safety and biological and clinical effects of autologous DCs modified with a nuclear factor $\kappa \mathrm{B}(\mathrm{NF}-\kappa \mathrm{B})$ inhibitor, exposed to a mixture of four citrullinated peptides, designated "Rheumavax", in a single-centre, open-labelled, first-in-human Phase I trial on RA patients (Benham et al. 2015). Vitamin D3-induced TolDC-MOG peptide treatment showed a beneficial effect in EAE, the mice model of MS, not only decreasing the incidence of the disease but also reducing the severity of the clinical signs mediated by induction of Tregs, as well as IL-10 production and reduction in Ag-specific lymphoproliferation. These results support VitD3-TolDC-peptide(s) treatment as a potential strategy to restore tolerance in autoimmune diseases such as MS (Mansilla et al. 2015). More recently, DCs differentiated with vitamin D3 alone or in combination with dexamethasone have been used in several clinical trials in patients with 
autoimmune diseases such as RA, type 1 diabetes, MS and Crohn's disease. The results are highly encouraging in terms of both safety and clinical efficacy in all the clinical studies completed to date; tolDC administration is tolerated and appears to be safe (Kim et al. 2019). However, numerous questions remain to be addressed prior to generalized and successful application of tolDCs in clinical settings. The major challenges are the optimization of the protocol to obtain a maximum number of tolDCs with stable tolerogenic properties and optimization of the dose, route and frequency of administration of each type of tolDC. As the successful ongoing clinical studies indicate, tolDC-based treatment of autoimmune diseases is now a reality and could provide innovative cellular therapy in future.

\section{Conclusion}

The enormous progress in our understanding of the pathogenesis and complexity of autoimmune diseases in recent years has led to the development of new in vivo and ex vivo approaches to induce antigen-specific tolerance. Antigenspecific approaches can only be achieved by active induction of immunoregulation by recruiting, eliciting and/or expanding autoantigen-specific regulatory $\mathrm{T}$ and $\mathrm{B}$ cells, in contrast to non-autoantigen-specific strategies that suppress inflammation, but also diminish protective immune response against microbial antigens. Antigen-specific therapies may enable restoration of immune homeostasis to the healthy steady state; thus, they may be curative, an outcome that non-antigen-specific approaches cannot deliver. Results obtained in Phase I/II trials demonstrated the safety and tolerability of these approaches. Phase II/III clinical trials will define whether the antigen-specific strategies would reach the goal of completely reversing the course of autoimmune diseases.

The results obtained thus far have underlined common requirements for achieving the effectiveness of the antigenspecific therapies including either peptide or protein delivery, antigen delivery via vehicles and regulatory cell-based approaches. The repetitive administrations of these and the use of multiple antigens are necessary to activate the tolerogenic arm of the immune response and to tackle the epitope spreading, respectively. The selection of the most suitable epitope/s to be used might be challenging, because different patients may display preferential response to specific antigens. Therefore, there is a huge need for the identification of antigenic peptides that can be used across different HLAtype patients.

Tolerogenic therapies should promote long-lasting effects, and this can be achieved by different mode of action, including the conversion of pathogenic Teff cells into Tregs or the de novo induction of Tregs. Modulated DC or APCs represent the population of cells able to prevent activation of pathogenic Teff cells, to promote de novo induction of Tregs and to re-educate Teff cells to become Tregs, thus maintaining long-term tolerance.

Several issues remain to be clarified for each of the approaches in the pipeline. Regardless of the tolerogenic approach used, one of the open questions in the field of tolerance induction is the definition of common parameters to monitor the response to treatment and to allow comparison of different approaches.

\section{Major current outstanding issues}

The main challenges for future research are as follows:

- Identification of autoantigens and hidden self-antigens

- Optimization of route of administration, dose and frequency of treatment

- Discovery of biomarkers for patient's stratification and therapeutic outcome

- Determination of the optimal disease stage for antigenbased therapies

- Development of the new generation of CAR-T cells, such as the universal chimeric antigen receptor (UniCAR)-T cells

- Development of donor-derived or third-party T cells that may be used to modularly generate CAR-T or CAR-Treg cell banks with batch production and improved safety.

\section{Where could we be in 5-10 years?}

It is expected that antigen-specific therapies for autoimmune diseases will continue to evolve. They will (1) incorporate new target specificities, (2) enhance efficacy and reduce adverse effects, and (3) gain acceptance as a first-line treatment option.

Antigen-specific therapeutics will offer patients the anticipated superior benefits of tissue- and disease-specific immunosuppressive effects that the monoclonal antibody revolution cannot deliver. We believe that the universal CAR-T cell system provides a direction for precision therapy to treat RA and can likely be applied to other systemic autoimmune diseases.

Cell-based personalized therapies might find their niche soon for the treatment of autoimmune diseases. However, their development will require substantial improvements to accelerate production, combine efficacy features, incorporate safety switches and reduce costs. It is expected that one or several of these strategies will succeed in delivering the long-awaited curative solution for patients with autoimmune diseases. 
Funding Open Access funding provided by Eötvös Loránd University. The funding was provided by the National Research Development and Innovation Office (Grant No. K 128546).

Open Access This article is licensed under a Creative Commons Attribution 4.0 International License, which permits use, sharing, adaptation, distribution and reproduction in any medium or format, as long as you give appropriate credit to the original author(s) and the source, provide a link to the Creative Commons licence, and indicate if changes were made. The images or other third party material in this article are included in the article's Creative Commons licence, unless indicated otherwise in a credit line to the material. If material is not included in the article's Creative Commons licence and your intended use is not permitted by statutory regulation or exceeds the permitted use, you will need to obtain permission directly from the copyright holder. To view a copy of this licence, visit http://creativecommons.org/licenses/by/4.0/.

\section{References}

Abbas AK, Trotta E, Simeonov R, Marson A, Bluestone JA (2019) Revisiting IL-2: biology and therapeutic prospects. Sci Immunol 3:eaat 1482. https://doi.org/10.1126/sciimmunol.aat1482

Alhadj Ali M, Liu YF, Arif S, Tatovic D, Shariff H, Gibson VB et al (2017) Metabolic and immune effects of immunotherapy with proinsulin peptide in human new-onset type 1 diabetes. Sci Transl Med 9:eaaf7779. https://doi.org/10.1126/scitranslmed.aaf7779

Andolfi G, Fousteri G, Rossetti M, Magnani CF, Jofra T, Locafaro G et al (2012) Enforced IL-10 expression confers type 1 regulatory T cell $(\operatorname{Tr} 1)$ phenotype and function to human CD4(+) T cells. Mol Ther 20:1778-1790. https://doi.org/10.1038/mt.2012.71

Bar-Or A, Vollmer T, Antel J, Arnold DL, Bodner CA, Campagnolo $D$ et al (2007) Induction of antigen-specific tolerance in multiple sclerosis after immunization with DNA encoding myelin basic protein in a randomized, placebo-controlled phase 1/2 trial. Arch Neurol 64:1407-1415

Battaglia M, Stabilini A, Draghici E, Migliavacca B, Gregori S, Bonifacio $E$ et al (2006) Induction of tolerance in type 1 diabetes via both CD4+CD25+ T regulatory cells and $\mathrm{T}$ regulatory type 1 cells. Diabetes 55:1571-1580. https://doi.org/10.2337/db05-1576

Benham H, Nel HJ, Law SC, Mehdi AM, Street S, Ramnoruth N et al (2015) Citrullinated peptide dendritic cell immunotherapy in HLA risk genotype-positive rheumatoid arthritis patients. Sci Transl Med 7:290ra87. https://doi.org/10.1126/scitranslmed.aaa9301

Bielekova B, Goodwin B, Richert N, Cortese I, Kondo T, Afshar G et al (2000) Encephalitogenic potential of the myelin basic protein peptide (amino acids 83-99) in multiple sclerosis: results of a phase II clinical trial with an altered peptide ligand. Nat Med 6:1167-1175. https://doi.org/10.1038/80516

Bonifacio E, Ziegler AG, Klingensmith G, Schober E, Bingley PJ, Rottenkolber $\mathrm{M}$ et al (2015) Effects of high-dose oral insulin on immune responses in children at high risk for type 1 diabetes: the Pre-POINT randomized clinical trial. JAMA 313:1541-1549. https://doi.org/10.1001/jama.2015.2928

Candia M, Kratzer B, Pickl WF (2016) On peptides and altered peptide ligands: from origin, mode of action and design to clinical application (immunotherapy). Int Arch Allergy Immunol 170:211-233. https://doi.org/10.1159/000448756

Chataway J, Martin K, Barrell K, Sharrack B, Stolt P, Wraith DC et al (2018) Effects of ATX-MS-1467 immunotherapy over 16 weeks in relapsing multiple sclerosis. Neurology 90:e955-e962. https:// doi.org/10.1212/WNL.0000000000005118
Clemente-Casares X, Blanco J, Ambalavanan P, Yamanouchi J, Singha $S$, Fandos $C$ et al (2016) Expanding antigen-specific regulatory networks to treat autoimmunity. Nature 530:434-440. https://doi. org/10.1038/nature1696

Cremel M, Guerin N, Horand F, Banz A, Godfrin Y (2013) Red blood cells as innovative antigen carrier to induce specific immune tolerance. Int J Pharm 443:39-49. https://doi.org/10.1016/j.ijpha rm.2012.12.044

Desreumaux P, Foussat A, Allez M, Beaugerie L, Hébuterne X, Bouhnik Y et al (2012) Safety and efficacy of antigen-specific regulatory T-cell therapy for patients with refractory Crohn's disease. Gastroenterology 143(1207-17):e1-2. https://doi.org/10.1053/j. gastro.2012.07.116

Ellebrecht CT, Bhoj VG, Nace A, Choi EJ, Mao X, Cho MJ, Di Zenzo G, Lanzavecchia A, Seykora Cotsarelis G, Milone MC, Payne AS (2016) Reengineering chimeric antigen receptor T cells for targeted therapy of autoimmune disease. Science 353(6295):179184. https://doi.org/10.1126/science.aaf6756

Feng X, Liu J, Xu W, Li G, Ding J (2020) Tackling autoimmunity with nanomedicines. Nanomedicine (Lond) 15(16):1585-1597

Ferreira LMR, Muller YD, Bluestone JA, Tang Q (2019) Next-generation regulatory T cell therapy. Nat Rev Drug Discov 18:749-769. https://doi.org/10.1038/s41573-019-0041-4

Fourlanos S, Perry C, Gellert SA, Martinuzzi E, Mallone R, Butler $\mathrm{J}$ et al (2011) Evidence that nasal insulin induces immune tolerance to insulin in adults with autoimmune diabetes. Diabetes 60:1237-1245. https://doi.org/10.2337/db10-1360

Fransson $\mathrm{M}$ et al (2012) CAR/FoxP3-engineered T regulatory cells target the CNS and suppress EAE upon intranasal delivery. J Neuroinfammation 9:112

Garren H (2008) Phase 2 trial of a DNA vaccine encoding myelin basic protein for multiple sclerosis. Ann Neurol 63:611-620

Gerace D, Martiniello-Wilks R, Simpson A (2015) Diabetes reversal via gene transfer: building on successes in animal models. Res Rep Endocrine Disorders 5:15-29. https://doi.org/10.2147/RRED. S51644

Getts DR, McCarthy DP, Miller SD (2013) Exploiting apoptosis for therapeutic tolerance induction. J Immunol 191:5341-5346. https ://doi.org/10.4049/jimmunol.1302070

Giannoukakis N, Phillips B, Finegold D, Harnaha J, Trucco M (2011) Phase I (safety) study of autologous tolerogenic dendritic cells in type 1 diabetic patients. Diabetes Care 34:2026-2032. https://doi. org/10.2337/dc11-0472

Goodnow GC, Sprent J, de St F, Groth B, Vinuesa CG (2005) Cellular and genetic mechanisms of self tolerance and autoimmunity. Nature 435(7042):590-597. https://doi.org/10.1038/nature03724

Gregori S, Roncarolo M-G (2018) Engineered T regulatory type 1 cells for clinical application. Front Immunol 9:233. https://doi. org/10.3389/fimmu.2018.00233

Hafler DA, Kent SC, Pietrusewicz MJ, Khoury SJ, Weiner HL, Fukaura H (1997) Oral administration of myelin induces antigen-specific TGF-beta 1 secreting T cells in patients with multiple sclerosis. Ann N Y Acad Sci 835:120-131. https://doi. org/10.1111/j.1749-6632.1997.tb48623.x

Hunter Z, McCarthy DP, Yap WT, Harp CT, Getts DR, Shea LD, Miller SD (2014) A biodegradable nanoparticle platform for the induction of antigen-specific immune tolerance for treatment of autoimmune disease. ACS Nano 8(3):2148-2160. https://doi. org/10.1021/nn405033r

Keeler GD, Kumar S, Palaschak B, Silverberg EL, Markusic DM, Jones NT et al (2018) Gene therapy-induced antigen-specific Tregs inhibit neuroinflammation and reverse disease in a mouse model of multiple sclerosis. Mol Ther 26:173-183. https://doi. org/10.1016/j.ymthe.2017.09.001 
Kim S-H, Jung H-H, Lee CK (2019) Generation, characteristics and clinical trials of ex vivo generated tolerogenic dendritic cells. Yonsei Med J 59(7):807-815. https://doi.org/10.3349/ ymj.2018.59.7.807

Kishimoto TK, Maldonado RA (2018) Nanoparticles for the induction of antigen-specific immunological tolerance. Front Immunol 9:230. https://doi.org/10.3389/fimmu.2018.00230

Lee J, Lunggren DK, Mao X, Manfredo-Vireira S, Nunez-Cruz S, Williams EF, Assenmacher CA, Radaelli E, Oh S, Wang B, Ellebrecht CT, Fraietta JA, Milone MC, Payne AS (2020) Antigen-specific B depletion for precision therapy of mucosal pemphigus vulgaris. J Clin Invest. https://doi.org/10.1172/JCI138416

Levings MK, Sangregorio R, Galbiati F, Squadrone S, de Waal MR, Roncarolo MG (2001) IFN-alpha and IL-10 induce the differentiationof human type $1 \mathrm{~T}$ regulatory cells. J Immunol 166:5530 5539. https://doi.org/10.4049/jimmunol.166.9.5530

Lin M, Stoica-Nazarov C, Surls J, Kehl M, Bona C, Olsen C et al (2010) Reversal of type 1 diabetes by a new MHC II-peptide chimera: "Single-epitope-mediated suppression" to stabilize a polyclonal autoimmune T-cell process. Eur J Immunol 40:2277-2288. https://doi.org/10.1002/eji.200940094

Liu J, Cao X (2015) Regulatory dendritic cells in autoimmunity: a comprehensive review. J Autoimmun 63:1-12. https://doi. org/10.1016/j.jaut.2015.07.011

Lutterotti A, Yousef S, Sputtek A, Sturner KH, Stellmann JP, Breiden P et al (2013) Antigen-specific tolerance by autologous myelin peptide-coupled cells: a phase 1 trial in multiple sclerosis. Sci Transl Med 5(188):188ra75. https://doi.org/10.1126/scitranslmed.3006168

Lutterotti A, Ludersdorfer TH, Docampo MJ, Hohmann M, Selles MC, Hayward-Koennecke $\mathrm{H}$ et al (2019) Establish tolerance in MS with myelin-peptide coupled red blood cells: the phase $\mathrm{Ib}$ ETIMSredtrial. ECTRIMS Online Library, Stockholm, p 279584

Mahmood Z, Schmalzing M, Dörner T, Tony HP, Muhammad K (2020) Therapeutic cytokine inhibition modulates activation and homing receptors of peripheral memory b cell subsets in rheumatoid arthritis patients. Front Immunol 11:572475. https://doi. org/10.3389/fimmu.2020.572475

Mansilla MJ, Sellès-Moreno C, Fàbregas-Puig S, Amoedo J, NavarroBarriuso J, Teniente-Serra A, Grau-López L, Ramo-Tello C, Martínez-Cáceres EM (2015) Beneficial effect of tolerogenic dendritic cells pulsed with MOG autoantigen in experimental autoimmune encephalomyelitis. CNS Neurosci Ther 21:222-230

Mauri C, Menon M (2017) (2017) Human regulatory B cells in health and disease: therapeutic potential. J Clin Invest 127(3):772-779. https://doi.org/10.1172/JCI85113

Nakayama M, Abiru N, Moriyama H, Babaya N, Liu E, Miao D et al (2005) Prime role for an insulin epitope in the development of type 1 diabetes in NOD mice. Nature 435:220-223. https://doi. org/10.1038/nature03523

Orban T, Farkas K, Jalahej H, Kis J, Treszl A, Falk B et al (2010) Autoantigen specific regulatory $\mathrm{T}$ cells induced in patients with type 1 diabetes mellitus by insulin B-chain immunotherapy. J Autoimmun 34:408-415. https://doi.org/10.1016/j. jaut.2009.10.005

Passerini L, Gregori S (2020) Induction of antigen-specific tolerance in T cell mediated diseases. Front Immunol 11:2194. https://doi. org/10.3389/fimmu.2020.02194

Pearson RM, Podojil JR, Shea LD, King NJC, Miller SD, Getts DR (2019) Overcoming challenges in treating autoimmunity: development of tolerogenic immune-modifying nanoparticles. Nanomedicine 18:282-291. https://doi.org/10.1016/j.nano.2018.10.00

Pozsgay J, Babos F, Uray K, Magyar A, Gyulai G, Kiss É, Nagy G, Rojkovich B, Hudecz F, Sármay G (2016) In vitro eradication of citrullinated protein specific B-lymphocytes of rheumatoid arthritis patients by targeted bifunctional nanoparticles. Arthritis Res Ther 18:15. https://doi.org/10.1186/s13075-016-0918-0

Pozsgay J, Szekanecz Z, Sarmay G (2017) Antigen specific immunotherapies in rheumatic diseases. Nat Rev Rheumatol 13:525-537. https://doi.org/10.1038/nrrheum.2017.107

Raker VK, Domogalla MP, Steinbrink K (2015) Tolerogenic dendritic cells for regulatory T cell induction in man. Front Immunol 6:569. https://doi.org/10.3389/fimmu.2015.00569

Roep BO (2013) Plasmid-encoded proinsulin preserves C-peptide while specifically reducing proinsulin-specific CD8+ T cells in type 1 diabetes. Sci Transl Med 5:191ra82. https://doi.org/10.1126/scitr anslmed.3006103

Romano M, Fanelli G, Albany CJ, Giganti G, Lombardi G (2019) Past, present, and future of regulatory $\mathrm{T}$ cell therapy in transplantation and autoimmunity. Front Immunol 10:43. https://doi.org/10.3389/ fimmu.2019.00043

Roncarolo MG, Gregori S, Bacchetta R, Battaglia M, Gagliani N (2018) The biology of T regulatory type 1 cells and their therapeutic application in immune-mediated diseases. Immunity 49:10041019. https://doi.org/10.1016/j.immuni.2018.12.001

Rosenblum MD, Gratz IK, Paw JS, Abbas AK (2012) Treating human autoimmunity: current practice and future prospects. Sci Transl Med 4(125):125sr1. https://doi.org/10.1126/scitranslmed.3003504

Serra P, Santamaria P (2019) Antigen-specific therapeutic approaches for autoimmunity. Nat Biotechnol 37(3):238-251. https://doi. org/10.1038/s41587-019-0015-4

Sharabi A, Tsokos MG, Ding Y, Malek TR, Klatzmann D, Tsokos GC (2018) Regulatory T cells in the treatment of disease. Nat Rev Drug Discov 17:823-844. https://doi.org/10.1038/nrd.2018.148

Sinha S, Subramanian S, Proctor TM, Kaler LJ, Grafe M, Dahan R et al (2007) A promising therapeutic approach for multiple sclerosis: recombinant Tcell receptor ligands modulate experimental autoimmune encephalomyelitis by reducing interleukin- 17 production and inhibiting migration of encephalitogenic cells into the CNS. J Neurosci 27:12531-12539. https://doi.org/10.1523/ JNEUROSCI.3599-07.2007

Ten Brinke A, Hilkens CM, Cools N, Geissler EK, Hutchinson JA, Lombardi $G$ et al (2015) Clinical use of tolerogenic dendritic cellsharmonization approach in European Collaborative Effort. Mediators Inflamm 2015:471719. https://doi.org/10.1155/2015/471719

Tsai S, Shameli A, Yamanouchi J, Clemente-Casares X, Wang J, Serra P, Yang Y, Medarova Z, Moore A, Santamaria P (2010) Reversal of autoimmunity by boosting memory-like autoregulatory $\mathrm{T}$ cells. Immunity 32:568-580

Vanderlugt CL, Miller SD (2002) Epitope spreading in immune-mediated diseases: implications for immunotherapy. Nat Rev Immunol 2:85-95. https://doi.org/10.1038/nri724

Walter M, Philotheou A, Bonnici F, Ziegler AG, Jimenez R (2009) No effect of the altered peptide ligand NBI-6024 on beta-cell residual function and insulin needs in new-onset type 1 diabetes. Diabetes Care 32:2036-2040. https://doi.org/10.2337/dc09-0449

Yoosuf S, Makharia GK (2019) Evolving therapy for celiac disease. Front Pediatr 7:193. https://doi.org/10.3389/fped.2019.00193

Zhang B, Wang Y, Yuan SJ, Liu L, Huang D, Hu J, Wang M, Li S, Song W, Chen H, Zhou D, Zhang X (2020) In vitro elimination of autoreactive B cells from rheumatoid arthritis patients by universal chimeric antigen receptor T cells. Ann Rheum Dis. https:// doi.org/10.1136/annrheumdis-2020-217844

Zhang Q, Lu W, Liang C-L, Chen Y, Liu H, Qiu F et al (2018) Chimeric antigen receptor (CAR) Treg: a promising approach to inducing immunological tolerance. Front Immunol 9:2359. https ://doi.org/10.3389/fimmu.2018.02359 